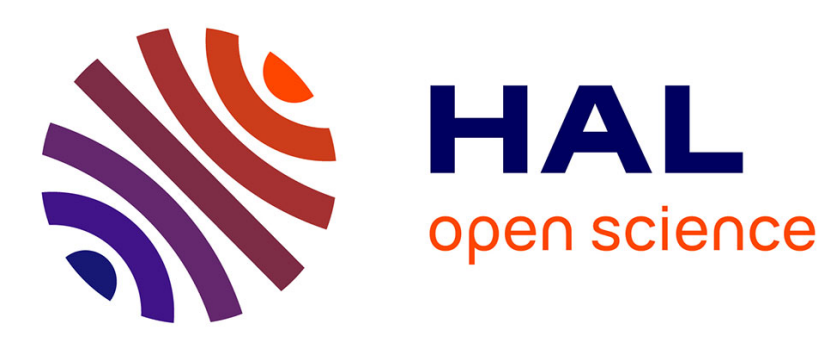

\title{
Effet de l'alimentation et de la race des vaches sur la fabrication de fromage d'Auvergne de Saint-Nectaire
} J.P. Garel, J.B. Coulon

\section{To cite this version:}

J.P. Garel, J.B. Coulon. Effet de l'alimentation et de la race des vaches sur la fabrication de fromage d'Auvergne de Saint-Nectaire. Productions Animales, 1990, 3 (2), pp.127-136. hal-00895895

\section{HAL Id: hal-00895895 \\ https://hal.science/hal-00895895}

Submitted on 1 Jan 1990

HAL is a multi-disciplinary open access archive for the deposit and dissemination of scientific research documents, whether they are published or not. The documents may come from teaching and research institutions in France or abroad, or from public or private research centers.
L'archive ouverte pluridisciplinaire HAL, est destinée au dépôt et à la diffusion de documents scientifiques de niveau recherche, publiés ou non, émanant des établissements d'enseignement et de recherche français ou étrangers, des laboratoires publics ou privés. 
INRA Prod. Anim., 1990, 3 (2), $127-136$

\section{J.P. GAREL, J.B. COULON*}

INRA Domaine expérimental de Marcenat 15190 Condat

* INRA Laboratoire de la Lactation

Theix, 63122 Saint-Genès Champanelle
Effet

de l'alimentation

et de la race

des vaches

sur la fabrication

de fromage

d'Auvergne

de Saint-Nectaire

Au cours des 10 dernières années, l'évolution rapide des facteurs de production et des contraintes de ramassage et de transformation du lait a entraîné des modifications de sa qualité avec des répercussions sur la quantité et la qualité des produits transformés. Ces modifications sont particulièrement importantes dans des régions où la majeure partie du lait est transformée en fromages, ce qui est notamment le cas de l’Auvergne.

\begin{abstract}
Si l'on connaît bien les principaux facteurs de variations de la composition chimique du lait (Hoden et al 1985, Journet et Chilliard 1985, Rémond 1985) et leurs interactions sur le terrain (EDE Haute-Saône 1985, Coulon et Binet 1987, Coulon et Lilas 1988, Froc et al 1988), les relations entre ces facteurs et l'aptitude fromagère du lait restent encore à préciser. Ainsi, l'effet de l'alimentation sur la fabrication du fromage a été essentiellement abordé par le biais des variations saisonnières (Chapman et Burnett 1972, Olson 1977, Grandison et al 1985); les études entreprises sur des effets directs de la ration et du génotype sont récentes et rares (Vertès et al 1989).
\end{abstract}

Résumé

Cette étude, composée de 4 essais successifs, avait pour but d'analyser les effets de l'alimentation (foin et ensilage d'herbe, herbe pâturée), et de la race (FFPN et Montbéliarde) sur les caractéristiques du fromage affiné. Le fromage fabriqué à l'échelle artisanale (100 litres de lait par fabrication) était le Saint Nectaire fermier, fromage d'Auvergne d'Appellation d'Origine Contrôlée.

Les différences de composition du lait entre races et régimes, essentiellement de taux protéique, se sont répercutées directement sur le rendement en fromage frais et affiné. Les rendements les plus élevés ont été obtenus avec les régimes à base de foin et d'herbe pâturée et avec les vaches Montbéliardes. La baisse du taux protéique du lait liée au régime ensilage d'herbe et à la race FFPN s'est traduite par une réduction du rendement fromager.

La qualité du fromage affiné a été faiblement influencée par la race. Le régime à base d'ensilage d'herbe n'a pas eu d'effets sur le goût des fromages. Il a modifié les caractéristiques des fromages affinés, en accentuant l'ouverture de la pâte et le gonflement précoce, mais en améliorant la souplesse et la couleur jaune des fromages.

Les critères simples retenus dans cette étude pour caractériser l'évolution du fromage, n'ont pas permis d'établir de liaisons entre la qualité du fromage frais et affiné, mais ont toutefois permis de mettre en évidence l'importance de la cave d'affinage.
L'objectif de cette étude, composée de 4 essais, a été d'analyser les effets de l'alimentation (type de ration de base) et de la race (Française Frisonne Pie Noire et Montbéliarde) sur les caractéristiques du fromage frais et affiné. Elle a été conduite en complément d'essais d'alimentation comparant, dans des conditions de montagne (Domaine INRA de Marcenat; $1100 \mathrm{~m}$ d'altitude), l'influence du type de ration de base hivernale et du niveau d'apport d'aliment concentré sur la production laitière, la composition du lait et la carrière des vaches.

\section{Conditions expérimentales}

\section{1 / Caractéristiques des régimes et des animaux}

Les 4 essais réalisés ont été décrits en détail par ailleurs (Dulphy et al 1984, Coulon et al 1985). Leurs principales caractéristiques sont présentées au tableau 1. Les essais 1, 2 et 3 ont concerné l'étude de l'effet du régime alimentaire hivernal (foin ou ensilage d'herbe) sur la qualité fromagère du lait, et l'essai 4, réalisé au pâturage, l'étude de l'effet de la race.

Dans les essais hivernaux $(1,2$ et 3$)$, les vaches laitières recevaient à volonté des rations à base de foin ou d'ensilage d'herbe de prairies naturelles, soit comme fourrage unique (essais 1 et 3 ) soit en ration mixte (foin + ensilage en proportion variable, essai 2). Ces rations de base étaient complémentées par un aliment de type production (1 UFL et $120 \mathrm{~g}$ PDI par $\mathrm{kg}$ brut) au delà d'une production de $7,5 \mathrm{~kg} / \mathrm{j}$ de lait en hiver (essais 1,2 et 3 ), et de $20 \mathrm{~kg} / \mathrm{j}$ au pâturage (essai 4). 
Tableau 1. Caractéristiques des laits utilisés pour la fabrication du fromage (moyenne des périodes de fabrications).

\begin{tabular}{|c|c|c|c|c|c|c|c|c|c|c|c|c|}
\hline \multirow[b]{2}{*}{ Essai } & & & \multicolumn{2}{|c|}{$\begin{array}{l}\text { Caractéristiques } \\
\text { des animaux }\end{array}$} & \multicolumn{6}{|c|}{ Caractéristiques des laits avant emprésurage } & \multirow{2}{*}{$\begin{array}{c}\text { Durée } \\
\text { affinage }\end{array}$} & \multirow{2}{*}{$\begin{array}{l}\text { Nombre } \\
\text { de } \\
\text { compa- } \\
\text { raisons }\end{array}$} \\
\hline & & & $\begin{array}{c}\text { Lait } \\
(\mathrm{kg} / \mathrm{i})\end{array}$ & $\begin{array}{l}\text { Stade de } \\
\text { lactation } \\
\quad \text { (j) }\end{array}$ & $\begin{array}{c}\text { Taux } \\
\text { butyreux } \\
(\mathrm{g} / \mathrm{kg})\end{array}$ & $\begin{array}{l}\text { Taux } \\
\text { protéique } \\
(\mathrm{g} / \mathrm{kg})\end{array}$ & $\underset{(\mathrm{g} / \mathrm{kg})}{\mathrm{MS}}$ & $\begin{array}{l}\text { Flore } \\
\text { totale } \\
(/ \mathrm{ml})\end{array}$ & $\begin{array}{c}\text { Bactéries } \\
\text { coliformes } \\
{[/ \mathrm{ml}]}\end{array}$ & $\begin{array}{l}\text { Contamin. } \\
\text { butyriques } \\
\text { des laits (1) }\end{array}$ & & \\
\hline 1 & $\begin{array}{l}\text { Effet régime } \\
1=\text { Foin } \\
2=\text { Ensilage d'herbe direct } \\
\text { Prairie naturelle }+ \\
\text { conservateur }(20 \% \mathrm{MS})\end{array}$ & $\begin{array}{l}\text { Hiver } 79 \\
\text { Hiver } 80\end{array}$ & $\begin{array}{l}16,0 \\
14,7\end{array}$ & $\begin{array}{l}122 \\
117\end{array}$ & $\begin{array}{l}33,8 \\
33,0\end{array}$ & $\begin{array}{l}30,1 \\
28,3\end{array}$ & $\begin{array}{l}116 \\
114\end{array}$ & $\begin{array}{l}252000 \\
363000\end{array}$ & $\begin{array}{l}213 \\
216\end{array}$ & $\begin{array}{l}60 \\
42\end{array}$ & $\begin{array}{c}3 \text { durées } \\
\text { d'affinage } \\
6-8-10 \text { sem. }\end{array}$ & 160 \\
\hline 2 & $\begin{array}{l}\text { Effet régime } \\
1=\text { Foin } 70 \%+30 \% \text { ensilage } \\
2=\text { Foin } 30 \%+70 \% \text { ensilage }\end{array}$ & Hiver 81 & $\begin{array}{l}17,3 \\
16,9\end{array}$ & $\begin{array}{l}122 \\
125\end{array}$ & $\begin{array}{l}31,6 \\
34,6\end{array}$ & $\begin{array}{l}28,5 \\
27,9\end{array}$ & $\begin{array}{l}115 \\
115\end{array}$ & $\begin{array}{l}165000 \\
159000\end{array}$ & $\begin{array}{l}68 \\
46\end{array}$ & $\begin{array}{l}93 \\
93\end{array}$ & $\begin{array}{l}1 \text { durée } \\
\text { d'affinage } \\
8 \text { sem. }\end{array}$ & 40 \\
\hline 3 & $\begin{array}{l}\text { Effet régime } \\
1=\text { Foin } \\
2=\text { Ensilage ressuvé }[27 \% \mathrm{MS}] \\
\text { Prairie naturelle } \\
\text { sans conservateur }\end{array}$ & Hiver 82 & $\begin{array}{l}20.8 \\
21.6\end{array}$ & $\begin{array}{l}65 \\
97\end{array}$ & $\begin{array}{l}31,2 \\
32,4\end{array}$ & $\begin{array}{l}29,3 \\
28,3\end{array}$ & $\begin{array}{l}112 \\
112\end{array}$ & $\begin{array}{l}135000 \\
117000\end{array}$ & $\begin{array}{l}48 \\
42\end{array}$ & $\begin{array}{l}54 \\
50\end{array}$ & $\begin{array}{c}1 \text { durée } \\
\text { d'affinage } \\
6 \text { sem. }\end{array}$ & 26 \\
\hline 4 & $\begin{array}{l}\text { Effet race } \\
1=\text { Montbéliarde } \\
2=\text { F.F.P.N. }\end{array}$ & $\begin{array}{l}\text { Elé } 79 \\
\text { Elé } 80\end{array}$ & $\begin{array}{l}14,8 \\
14,6\end{array}$ & $\begin{array}{l}179 \\
194\end{array}$ & $\begin{array}{l}36,3 \\
37,2\end{array}$ & $\begin{array}{l}32,4 \\
31,7\end{array}$ & $\begin{array}{l}124 \\
123\end{array}$ & $\begin{array}{l}211000 \\
222000\end{array}$ & $\begin{array}{l}235 \\
299\end{array}$ & $\begin{array}{l}83 \\
82\end{array}$ & $\begin{array}{c}3 \text { durées } \\
\text { d'affinage } \\
6-8-10 \text { sem. }\end{array}$ & 135 \\
\hline
\end{tabular}

(1) Pourcenlage de lait avec moins de 1000 spores/l. (Contamination $>10000$ spores/l:7\% dans l'essai 1 . Nulle dans les autres essais).

Les animaux, qui vêlaient en majorité en début d'hiver, étaient conduits, du début novembre au début mai, en stabulation entravée. La traite avait lieu en place, au pot. Dans l'essai 4, réalisé au pâturage, les animaux des 2 races étaient conduits ensemble, en un seul troupeau, en pâturage tournant avec un chargement fort au printemps de 3 vaches/ha et une fertilisation de 80 unités d'azote/ha.

\section{2 / Méthode de fabrication du fromage}

Le fromage fabriqué dans cette étude a été le Saint Nectaire, fromage d'Auvergne d'A ppellation d'Origine Contrôlée. Ce fromage à pâte pressée, fabriqué à partir du lait cru, est de taille moyenne (1,7 kg environ). Sa production est en grande partie d'origine fermière.

La méthode de fabrication a été de type fermier avec du lait cru, selon les conditions d'appellation d'origine, (communication du Syndicat de St Nectaire, M. Verlaguet). Chaque jour,

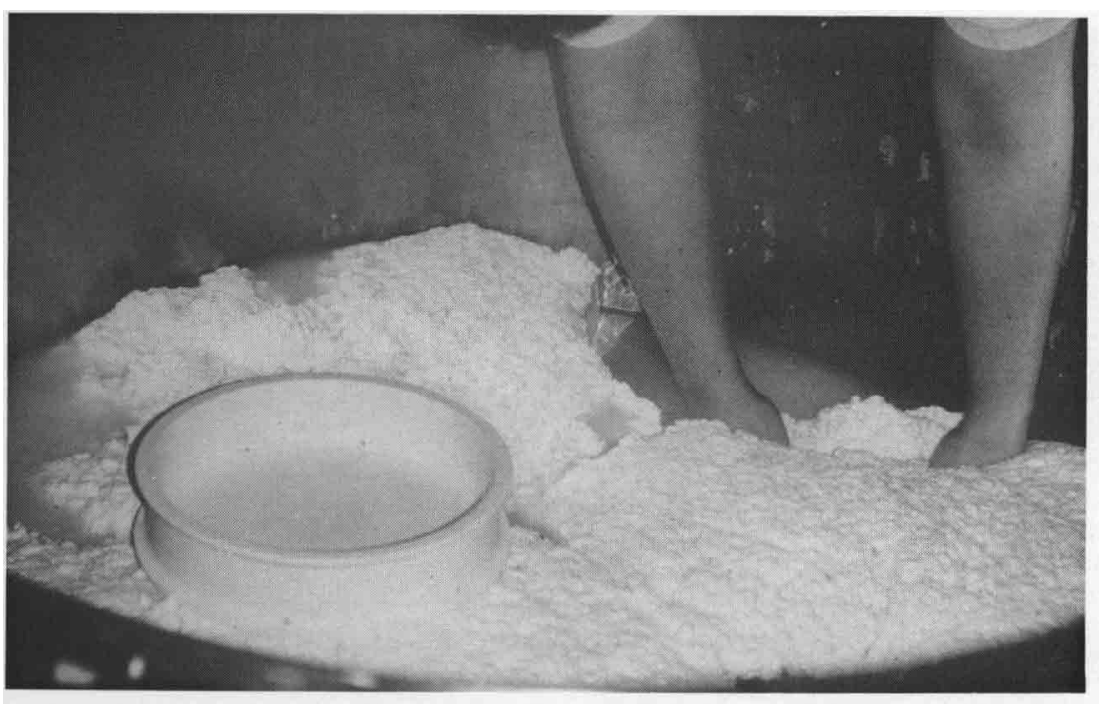

2 traitements expérimentaux ont été comparés, en réalisant en parallèle 2 fabrications de fromage avec 100 litres de lait chacune.

Le lait de chaque fabrication, issu de la traite du matin provenait du lait de 10 à 12 vaches, en majorité multipares, indemnes de mammites. Dans les essais hivernaux elles étaient pour moitié de race FFPN et pour l'autre de race Montbéliarde. Chaque essai comprenait plusieurs périodes ( 4 à 5 dans les essais 1 et 4 , 2 dans les essais 2 et 3 ) d'une durée de 3 semaines chacune, espacées d'une dizaine de jours. Chaque cuve de fabrication en bois (gerle) était affectée à un type de lait, et celà au cours de tout l'essai. Ainsi, avant le début des essais et aux inter-périodes, chaque cuve était rincée avec du lait correspondant à chaque lot expérimental.

Le caillage se faisait à une température de $33^{\circ} \mathrm{C}$ avec un léger réchauffement du lait, de l'ordre de 1 à $2^{\prime \prime} \mathrm{C}$, par circulation d'eau chaude dans un serpentin immergé dans le lait. La température de la salle de fabrication était maintenue à $15^{\circ} \mathrm{C}$. La quantité de présure n'a pas varié au cours de tout l'essai $(40 \mathrm{ml}$ pour 100 litres de lait). A près coagulation totale ( 1 heure environ) le sérum était soutiré, et le caillé était découpé avant d'être mis en moule. Le pressage se faisait avec une machine de type DUPRAT avec réchauffement du caillé par bain d'huile. Les fromages, au nombre de 5 à 6 par fabrication, étaient mis sous presse jusqu'au lendemain. Le salage de surface, réalisé 5 heures environ après le moulage, a été de $2 \mathrm{~g}$ de sel/ $100 \mathrm{~g}$ de caillé en hiver et de $2,5 \mathrm{~g}$ en été.

Tous les fromages ont été mis à sécher dans un local aéré à une température constante de $10^{\circ} \mathrm{C}$ pendant 4 à 5 jours avant la mise en cave. L'affinage a été confié à un seul affineur pendant toute la durée de l'essai, excepté dans l'essai 3 où deux caves d'affinage ont été comparées. 
Dans ces conditions, seuls un à deux fromages par fabrication ont été suivis au cours de l'affinage. Dans les essais 1 et 4, l'effet de la durée de l'affinage a été testé $(6,8$ et 10 semaines). Une seule durée d'affinage a été retenue dans l'essai 2 (8 semaines) et dans l'essai 3 ( 6 semaines).

\section{3 / Mesures}

Les mesures sur la production laitière, la composition du lait, l'alimentation et la santé des animaux ont été réalisées individuellement (Dulphy et al 1984, Coulon et al 1985). A l'échelle du troupeau, la flore totale a été mesurée sur l'ensemble du lait refroidi.

Sur le lait de mélange, un échantillon de lait a été prélevé dans la cuve de fabrication, 2 heures environ après son remplissage. Les analyses suivantes ont été réalisées: matière sèche et taux butyreux (tous les jours), taux protéique (1 fois par semaine en 1979 puis tous les jours), flore totale, butyrique et coliforme ( 2 fois par semaine).

Le temps de coagulation du lait a été apprécié par observation visuelle de l'état du lait dans la cuve de fabrication. Sur les fromages, le poids, les teneurs en matière sèche et en matière grasse ont été mesurés au moulage, à 24 heures ot après affinage.

La qualité des fromages a été appréciée en 2 temps : avant la mise en cave sur l'ensemble des fromages (notation des gonflements éventuels) et en fin d'affinage (notation de l'aspect et du goût). Les échelles de notation sont présentées en annexe 1 et sur la figure 1. En 1979 et 1980 , les critères d'appréciations tenaient compte de la forme, de la pâte (ouverture, souplesse et couleur) et du goût. A partir de 1981, on a adopté la grille de classement proposée par le Syndicat de Contrôle du Saint Nectaire. Cette grille retient 3 critères : la forme, la pâte et le goût. Elle définit le fromage de bonne qualité de la façon suivante: une forme régulière, une pâte souple, onctueuse pouvant présenter quelques ouvertures, une coloration homogène, un goût franc et savoureux pouvant être légèrement lactique avec une odeur agréable.
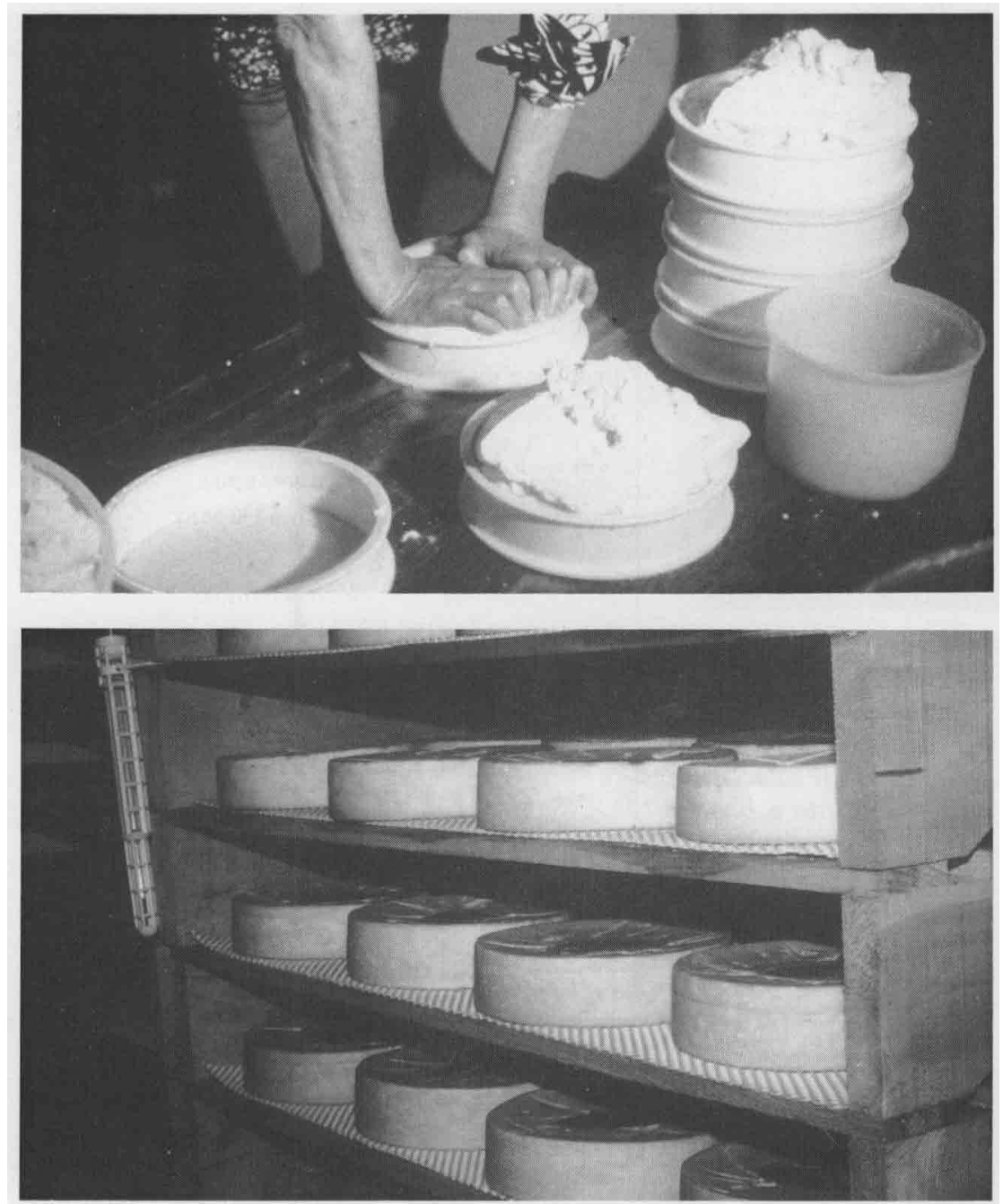

La production de Saint-Nectaire, fromage à pâte pressée, est en grande partie d'origine fermière. Le caillé est mis en moule, pressé puis salé. Après quelques jours de séchage, les fromages sont affinés en caves pendant plusieurs semaines.

En l'absence du jury de dégustation, les qualités organoleptiques ont été appréciées par un groupe de 3 personnes de l'INRA et un professionnel du Syndicat de St Nectaire.

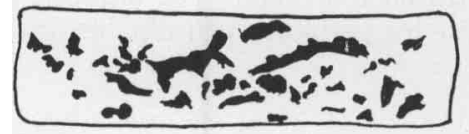

de trous" : na pas ete rencontrée dans cette étude).
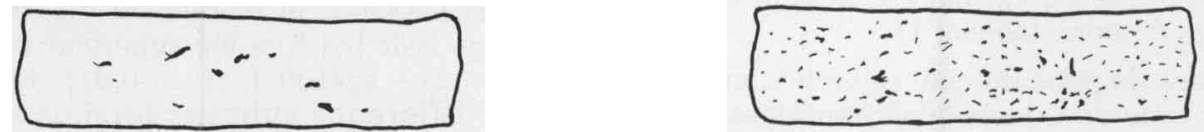
Tableau 2. Influence du régime hivernal sur la fabrication fromagère.

\section{Le rendement en caillé et en fromage affiné est plus élevé avec un régime à base de foin qu'avec un régime à base d'ensilage d'herbe.}

\begin{tabular}{|c|c|c|}
\hline & \multicolumn{2}{|c|}{ Essai 1} \\
\hline & Foin & Ensilage \\
\hline Temps de prise du lait (min) & 10,6 & 9,6 \\
\hline \multicolumn{3}{|l|}{ Evolution du taux de MS \% } \\
\hline - au moulage & 47,8 & 47,9 \\
\hline - à $24 \mathrm{~h}$ & 50,3 & 50,5 \\
\hline - à 6 semaines & 55,0 & 55,0 \\
\hline - à 8 semaines & 57,1 & 57,4 \\
\hline - à 10 semaines & 58,4 & 59,4 \\
\hline \multicolumn{3}{|l|}{ Evolution du gras/sec } \\
\hline- à $24 \mathrm{~h}$ & 51,5 & 51,5 \\
\hline - à 6 semaines & 53,6 & 54,6 \\
\hline - à 8 semaines & 53,8 & 54,2 \\
\hline - à 10 semaines & 54,1 & 54,3 \\
\hline \multicolumn{3}{|l|}{ Taux de sel du fromage affiné ( $\%$ MS) } \\
\hline - à 6 semaines & 1,03 & 1,00 \\
\hline - à 8 semaines & 0,99 & 1,01 \\
\hline - à 10 semaines & 1,06 & 1,03 \\
\hline \multicolumn{3}{|l|}{ Rendement kg/100 I de lait } \\
\hline - en caillé & 18,0 & 17,1 \\
\hline - en fromage frais à $24 \mathrm{~h}$ & 11,4 & 10,7 \\
\hline - en MS à 24 h & 5,7 & 5,4 \\
\hline $\begin{array}{r}\text { - en tromage affine } \\
\text { à } 6 \text { semaines }\end{array}$ & 10,2 & 9,5 \\
\hline à 8 semaines & 9,9 & 9,3 \\
\hline à 10 semaines & 9,4 & 9,0 \\
\hline \multicolumn{3}{|l|}{ Défauts (\% de l'effectif) } \\
\hline - de forme & 15 & 13 \\
\hline - de pâte (dureté) & 18 & 10 \\
\hline - d'ouverture & 22 & 29 \\
\hline - de goût & 16 & 13 \\
\hline Coloration jaune (en \%) & 14 & 89 \\
\hline
\end{tabular}

\section{4 / Interprétation des résultats}

Les résultats portent sur 720 fabrications (soit 820 fromages affinés) réparties sur 4 années. Les effets des traitements expérimentaux ont été étudiés essai par essai. Les critères de rendement, exprimés en $\mathrm{kg} / 100$ litres de lait ont été les suivants : rendements en caillé, en fromage frais et en fromage affiné. Pour tenir compte des variations de taux de matière sèche du fromage, le critère de rendement en fromage frais a été exprimé en quantité de matière sèche.

Le mode d'appréciation de la qualité des fromages utilisé sur l'ensemble de l'étude n'a permis de mettre en évidence que les défauts (forme, pâte et goût).

Le traitement statistique des résultats a été réalisé par analyse de variance. Les facteurs suivants ont été pris en compte : traitement (alimentaire ou racial), année, période de fabrication, et durée d'affinage. Par ailleurs, les relations entre les variables principales ont été analysées.

\section{Résultats}

\section{1 / Effet de la nature de la ration de base}

Dans les 3 essais hivernaux, le taux protéique du lait a toujours été plus faible (de respectivement $1,8,0,6$ et $1 \mathrm{~g} / \mathrm{kg}$ ) pour les régimes à base d'ensilage d'herbe (tableau 1).

Le temps de prise du caillé a varié d'un essai à l'autre avec une durée plus importante dans
Figure 2. Temps de prise du lait (moyenne/période)

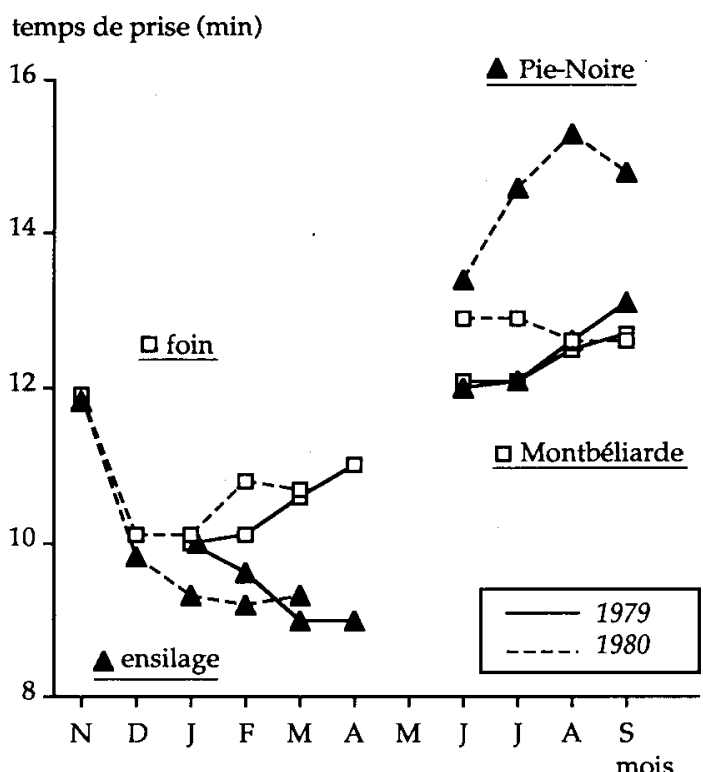

l'essai 2. La nature du régime n'a eu un effet marqué qu'en 2ème partie de l'essai 1, le régime à base d'ensilage d'herbe ayant réduit de façon significative $(-1 \mathrm{mn}, \mathrm{p}<0,01\}$ le temps de prise du lait (figure 2).

Dans l'essai 1, le rendement en caillé et en fromage frais $(24 \mathrm{~h}$ ) a été supérieur pour le lot foin ( $+0,7 \mathrm{~kg} / 100 \mathrm{l}, \mathrm{p}<0,01$ ) (tableau 2). Cette différence subsiste lorsque l'on tient 
Tableau 3. Influence de la proportion et du type d'ensilage d'herbe sur la fabrication fromagère.

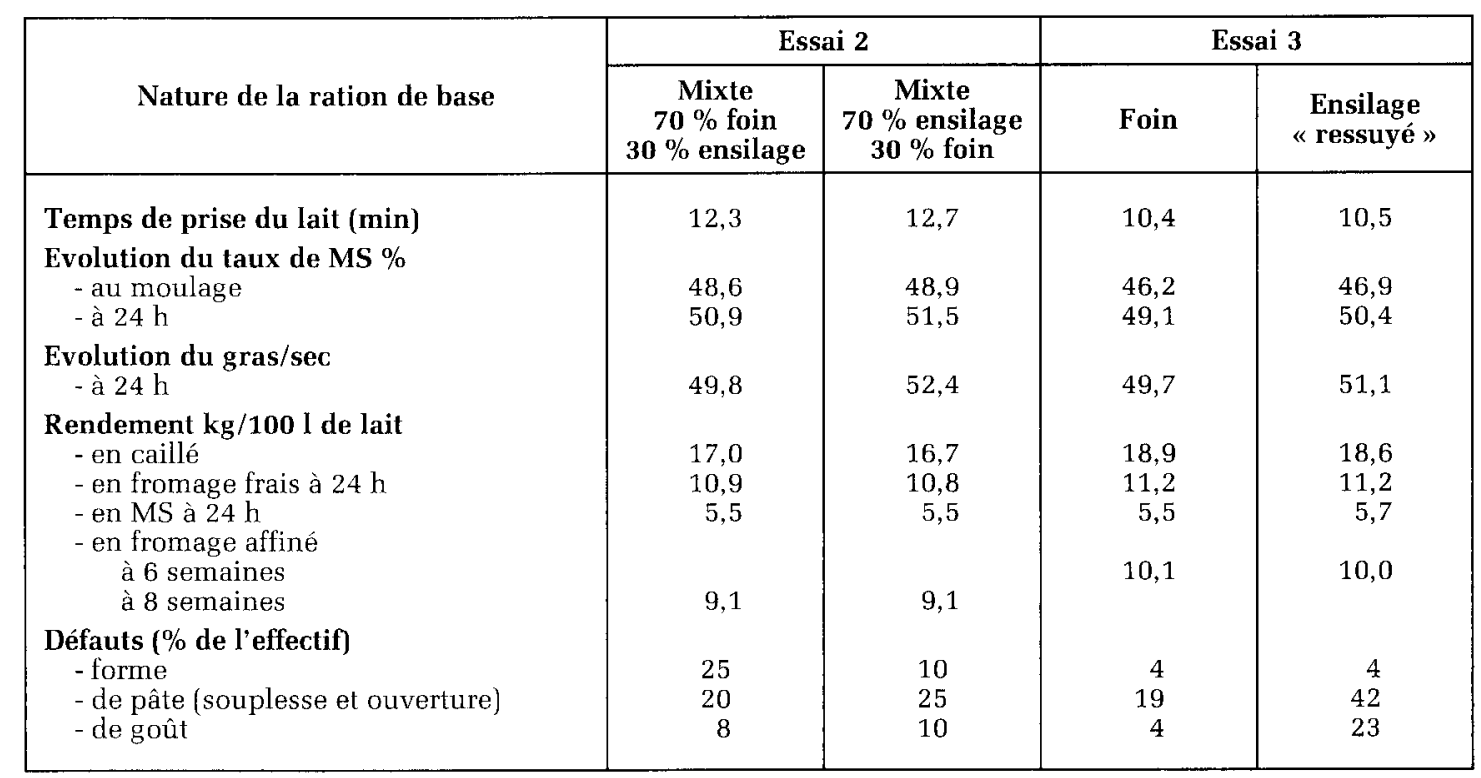

compte de l'écart de taux protéique entre les 2 lots (+ 0,3 kg/100 l, p $<0,01$ ), (qui présentaient par ailleurs des taux butyreux voisins). Le rendement en fromage (affiné pendant 8 semaines) a été supérieur de $0,6 \mathrm{~kg} / 100 \mathrm{l}$ ( $\mathrm{p}<$ 0,01 ) pour le lot foin.

Dans les essais 2 et 3 , l'effet du régime alimentaire sur les rendements (caillé, fromage frais et affiné) a été peu marqué (tableau 3). Les défauts des fromages affinés semblent cependant en moyenne plus nombreux avec les régimes à forte proportion d'ensilage d'herbe.

Sur l'ensemble des fromages fabriqués (685 pour le foin et 702 pour l'ensilage), la proportion de fromages ayant présenté des gonflements précoces a été plus forte pour le régime ensilage $(10 \%)$ que pour le régime foin (5\%). Il n'y a pas eu d'incidence du régime dans les essais 2 et 3 . L'introduction de l'ensilage d'herbe dans la ration a eu pour effet d'augmenter le degré d'ouverture de la pâte du fromage (tableau 2), d'accentuer sa coloration jaune et d'assouplir sa pâte. Le goût du fromage, en revanche, ne semble pas avoir été affecté par le régime, si ce n'est dans l'essai 3 (avec toutefois un nombre de fromages limité). Il existe cependant une grande variabilité d'une fabrication à l'autre pour ces critères de qualité de fromage. Il n'est pas apparu d'interactions entre le régime et la durée d'affinage.

L'effet du pâturage a été estimé en comparant les fabrications réalisées aux périodes hivernales et estivales (au printemps en comparant les fabrications d'avril et de juin, et à l'automne en comparant celles d'octobre et de décembre).

Au printemps comme à l'automne, le pâturage s'est accompagné d'une augmentation du rendement en caillé et en fromage frais par rapport à l'alimentation hivernale (figure 3). Cette augmentation de rendement est surtout importante lors du passage du régime ensilage au régime d'herbe ( +7 à $12 \%$ ) en liaison avec l'augmentation des taux protéiques et butyreux observés lors de ces changements de régime.

\section{2 / Effet de la race}

Les vaches Montbéliardes ont produit un lait plus riche en matière protéique $(+0,7 \mathrm{~g} / \mathrm{kg})$, mais plus pauvre en matière grasse que celui des vaches Frisonnes $(-0,9 \mathrm{~g} / \mathrm{kg})$.

Le temps de prise du lait a été comparable durant l'été 1979 , et supérieur pour les Frisonnes durant l'été 1980 (figure 2).

Les rendements en caillé et en fromage frais (tableau 4) ont été plus élevés chez les vaches Montbéliardes que chez les vaches Frisonnes (respectivement $+0,6$ et $0,3 \mathrm{~kg} / 1001, \mathrm{p}<$ $0,01)$. Malgré un taux de MS du fromage plus élevé chez les Frisonnes, le rendement en fromage frais exprimé en MS est resté supérieur chez les Montbéliardes (+ 0,2 kg/100 l).

Figure 3. Evolution du rendement en caillé à la mise à l'herbe et à la rentrée à l'étable.

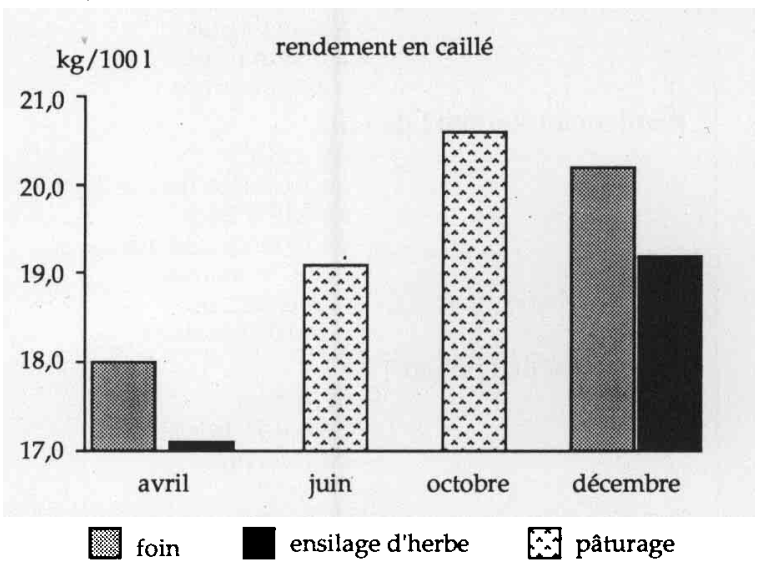


Sur l'ensemble des fromages frais $(700$ fromages par race), le pourcentage de gonflements précoces a été faible ( $2 \%$ ), et identique pour les 2 races. Les fromages affinés provenant des vaches Montbéliardes ont eu tendance à être plus durs que ceux des vaches Frisonnes (31\% de défauts contre $13 \%$, avec un taux de matières grasses des fromages inférieur (de 1 à $2 \%$ de gras/sec par rapport aux Frisonnes). Il n'est pas apparu de différences significatives sur les autres critères.

\section{3 / Liaison entre la composition du lait et la fabrication fromagère}

Pour étudier les relations entre la composition du lait et la qualité des fromages, toutes les fabrications réalisées ont été retenues. Toutefois, certaines mesures n'ayant pas été effectuées dans tous les essais, les effectifs varient selon les liaisons étudiées.

\section{1 / Influence du taux de matière sèche du lait}

Chaque année, le taux de matière sèche du lait a varié de façon importante au cours de la saison. Il a été plus faible en hiver qu'en été ( $115 \mathrm{~g} / 1000$ en hiver et $123 \mathrm{~g} / 1000$ en été). Les variations journalières du taux de MS du lait au cours d'une période de fabrication de 3 semaines ont été plus accentuées au pâturage (coefficient de variation journalière (cv) de $3,7 \%$ ) que sur le régime hivernal ( $\mathrm{cv}=2 \%$ ), en raison essentiellement du mode d'exploitation du pâturage en rotation (figure 4).
Les taux de matière sèche du caillé et du fromage frais ont présenté des variations beaucoup plus faibles mais encore sensibles (figure 4). Elles paraissent largement indépendantes des variations de la teneur en MS du lait.

Si les variations journalières de taux de MS ont été fortement tamponnées dès le moulage, il existe cependant des écarts importants au sein d'une même fabrication. Sur les 2 fromages retenus pour l'affinage dans une même fabrication, la moyenne des écarts absolus entre les 2 fromages a été de 1,3 point de MS au moulage et de 0,9 point de MS à $24 \mathrm{~h}$.

\section{2 / Influence de la composition chimique du lait}

Le rendement en fromage à $24 \mathrm{~h}$ (exprimé en $\mathrm{kg} \mathrm{MS/100} \mathrm{l} \mathrm{de} \mathrm{lait),} \mathrm{a} \mathrm{varié} \mathrm{de} \mathrm{5,2} \mathrm{à} \mathrm{6,8} \mathrm{selon}$ la saison (figure 5). Cette variation de rendement est en grande partie liée à celle du taux de matière utile : $0,09 \mathrm{~kg}$ de fromage par point de matière utile supplémentaire $(r=0,86)$, avec une influence du taux protéique 2 fois plus importante que celle du taux butyreux, selon l'équation :

$\mathrm{y}=0,06 \mathrm{~TB}+0,13 \mathrm{TP}-0,23 \pm 0,25$

$r=0,87 \mathrm{n}=317$

où y représente le rendement en fromage à $24 \mathrm{~h}$ (exprimé en $\mathrm{kg} \mathrm{MS/100} \mathrm{l} \mathrm{de} \mathrm{lait)} \mathrm{et} \mathrm{où} \mathrm{les} \mathrm{taux}$ sont exprimés en $\mathrm{g} / \mathrm{kg}$ de lait.

L'influence du taux protéique est apparue importante quel que soit le rendement considéré (fromage frais ou fromage affiné). Il est

Tableau 4.Influence de la race sur la fabrication fromagère: comparaison entre FFPN et Montbéliarde au pâturage (essai 4).

\section{Les rendements en fromage des Montbéliardes sont supérieurs à ceux des Frisonnes.}

\begin{tabular}{|c|c|c|}
\hline & MO & FFPN \\
\hline Temps de prise du lait (min) & 12,5 & 13,4 \\
\hline \multicolumn{3}{|l|}{ Evolution du taux MS \% } \\
\hline - au moulage & 47,7 & 48,3 \\
\hline - à $24 \mathrm{~h}$ & 51,1 & 51,5 \\
\hline - à 6 semaines & 58,4 & 59,4 \\
\hline - à 8 semaines & 59,5 & 60,3 \\
\hline - à 10 semaines & 61,2 & 61,7 \\
\hline \multicolumn{3}{|l|}{ Evolution du gras/sec } \\
\hline$-\grave{a} 24 \mathrm{~h}$ & 50,3 & 51,7 \\
\hline - à 6 semaines & 51,3 & 53,5 \\
\hline - à 8 semaines & 52,3 & 53,4 \\
\hline - à 10 semaines & 51,8 & 53,9 \\
\hline \multicolumn{3}{|l|}{ Taux de sel du fromage affiné (\% MS) } \\
\hline - à 6 semaines & 1,56 & 1,57 \\
\hline - à 8 semaines & 1,58 & 1,63 \\
\hline - à 10 semaines & 1,67 & 1,75 \\
\hline \multicolumn{3}{|l|}{ Rendement $\mathrm{kg} / 100$ I de lait } \\
\hline- en caillé & 19,7 & 19,1 \\
\hline - en fromage frais à $24 \mathrm{~h}$ & 12,4 & 12,1 \\
\hline - en MS à $24 \mathrm{~h}$ & 6,4 & 6,2 \\
\hline - en fromage affiné & 10.7 & 10.4 \\
\hline à 8 semaines & 10,4 & 10,1 \\
\hline à 10 semaines & 10,1 & 9,9 \\
\hline \multicolumn{3}{|l|}{ Défauts (\% de l'effectif) } \\
\hline- de forme & 8 & 11 \\
\hline - de pâte (dureté) & 31 & 13 \\
\hline - d'ouverture & 20 & 25 \\
\hline - de goût & 18 & 20 \\
\hline Coloration jaune (en \%) & 71 & 89 \\
\hline
\end{tabular}


Figure 4. Variations journalières du taux de M.S. du lait et du fromage.

taux de MS (\%)

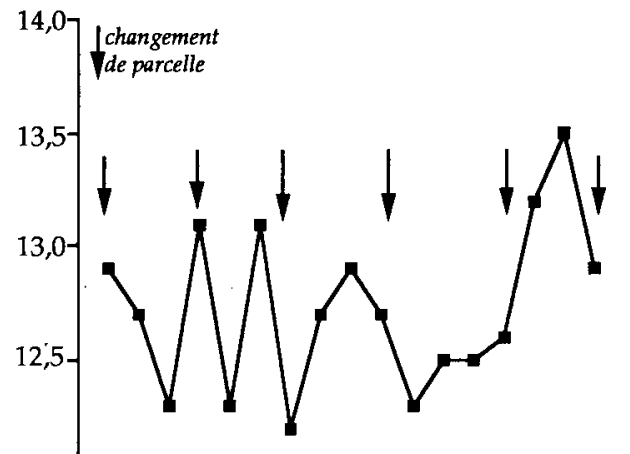

12,0
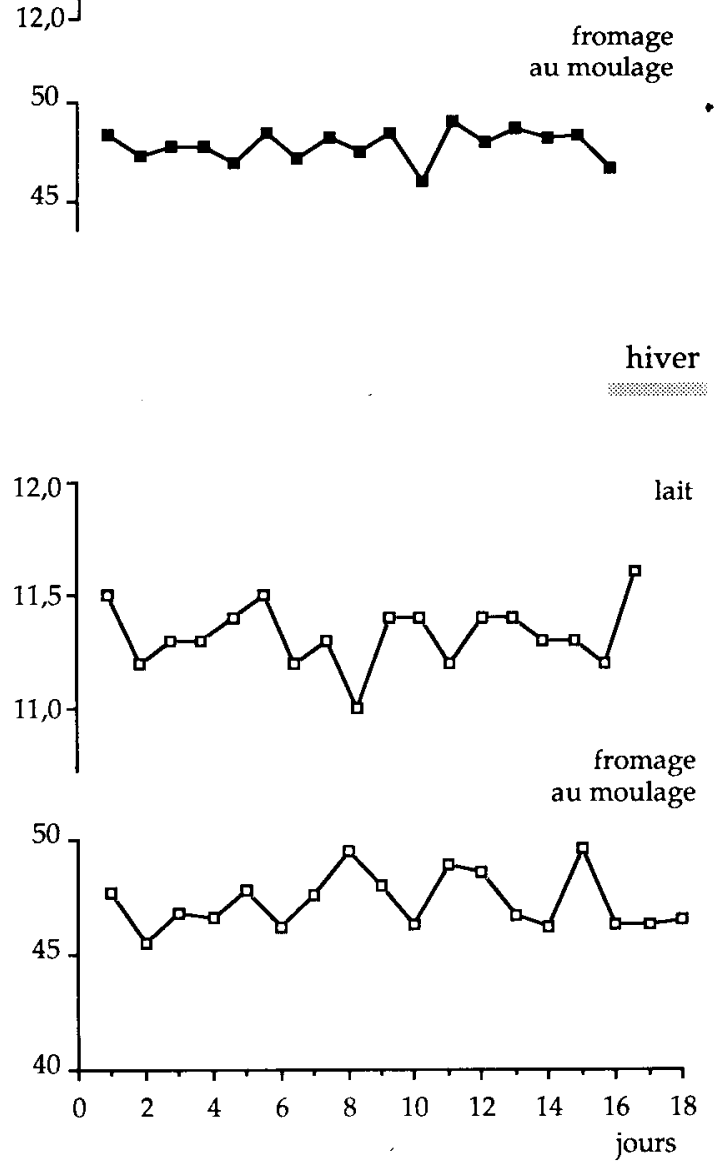

cependant difficile de dissocier les effets respectifs du taux protéique et du taux butyreux. Même si la composition du lait a été différente entre les essais réalisés sur régime hivernal ou au pâturage, les liaisons entre le rendement et le taux protéique ont été semblables (figure 6).

A l'intérieur de chaque type de régime (régime à base de fourrages conservés et régime à base d'herbe pâturée), les qualités du fromage se sont améliorées lorsque les laits étaient plus riches en matières protéiques et en matières grasses. Les défauts les plus graves sont apparus vers la fin de l'hiver (en mars) et au début du pâturage (en juin et juillet ; figure 7). Toutefois, il est possible que d'autres facteurs soient intervenus à ces périodes, en particulier au cours de la fabrication et de l'affinage.

\section{3 / Influence de la flore du lait}

La flore totale du lait dans la cuve de fabrication a été plus élevée les 2 premières années que les années suivantes. Ces variations sont à mettre en parallèle avec les résultats bactériologiques observées à l'échelle du troupeau sur le lait refroidi en tank: 120000 germes/ml dans l'essai 1 contre 17000 et 37000 germes $/ \mathrm{ml}$ dans les essais 2 et 3. L'augmentation de la flore du lait n'a pas entraîné de différences sensibles dans la fabrication du fromage. L'augmentation de la contamination du lait par la flore butyrique (entre 1000 spores/l et 10000 spores/l) n'a pas augmenté de façon sensible les défauts importants d'ouverture du fromage. Toutefois, les fromages à faible ouverture sont plus nombreux (37\% contre $26 \%$ ) lorsque la contamination butyrique est faible (figure 8 ).

Figure 5. Evolution du rendement fromager au cours de l'année.

rendement fromager ( $\mathrm{kg} \mathrm{MS/} 100 \mathrm{l})$

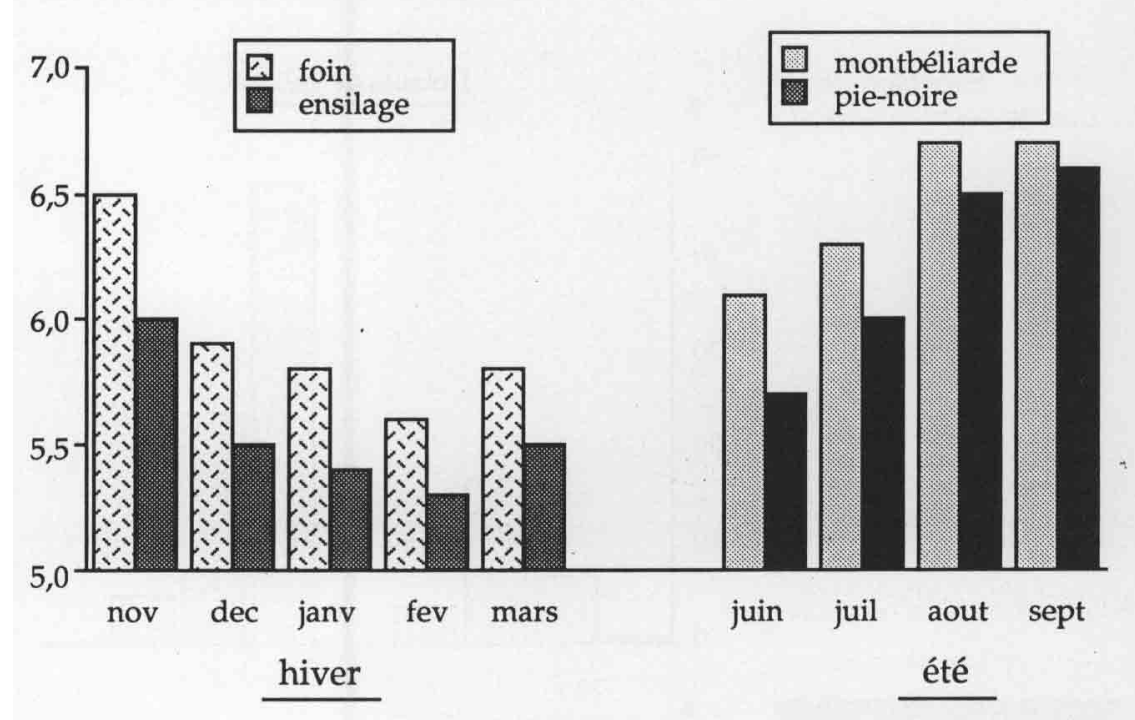

Figure 6. Liaison entre le rendement en fromage frais et le taux protéique.

rendement (kg MS/ $100 \mathrm{l}$ )

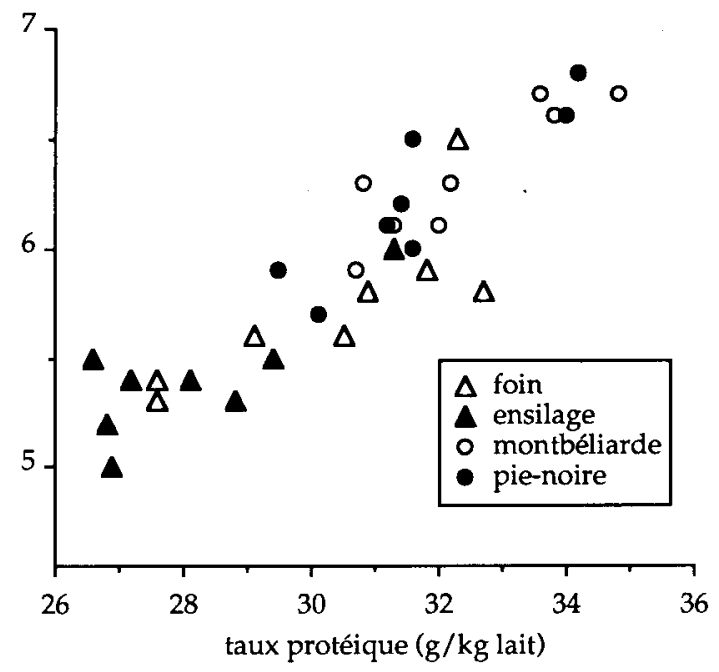




\section{4 / Influence de l'affinage}

Les critères de rendement, les taux de MS et de MG ont été étroitement liés entre le fromage frais et le fromage affiné (coefficient de corrélation de 0,97 entre le rendement en fromage frais et affiné). Un accroissement de $1 \%$ du taux de MS du fromage frais s'est traduit par un accroissement de $0,8 \%$ pour le fromage affiné ; l'influence du taux de matière grasse (exprimé en \% de MS) a été moindre $(0,5 \%)$. L'allongement de la durée d'affinage de 6 à 10 semaines s'est accompagné d'une augmentation du taux de MS de 3,4\% ( $p<0,01$ ), sans modifier le taux de MG dans la MS.

Dans cette étude, la qualité du fromage affiné n'a pas pu être reliée aux critères simples mesurés sur le fromage frais. Seul l'effet du mode d'affinage (comparaison de 2 caves d'affinage dans l'essai 3) a eu une influence significative sur la forme $(0,8$ point $/ 5)$ sur la pâte $(1,4$ point $/ 5$ ) et le goût $(1,5$ point $/ 10)$, avec toutefois un nombre réduit de fromages (26 fromages/ cave).

Figure 7. Evolution de la qualité selon la saison.
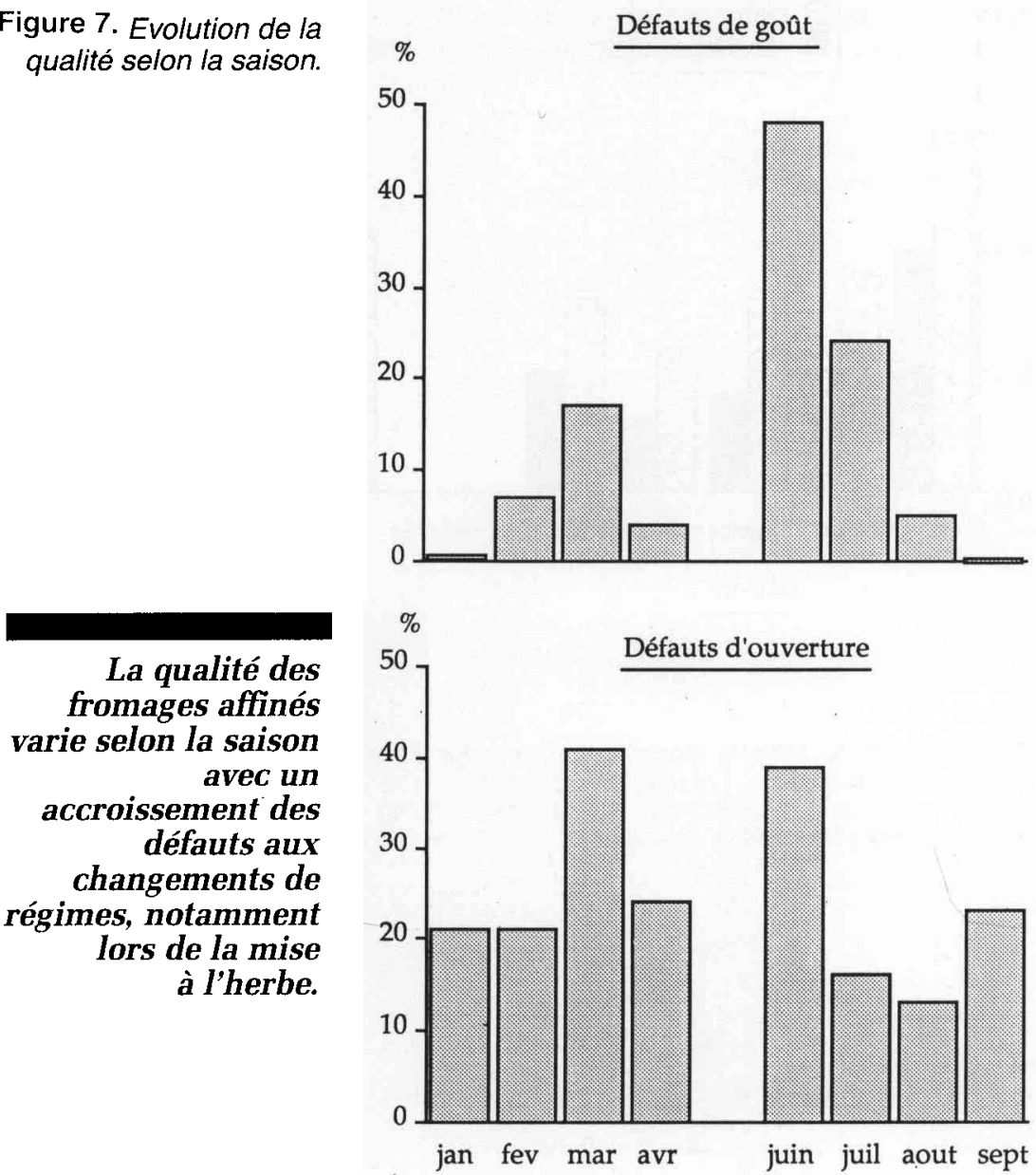

\section{Discussion - Conclusion}

Cette étude confirme l'influence des facteurs zootechniques sur la composition du lait. Les rations à base d'ensilage d'herbe ont entraîné une réduction des teneurs en protéines du lait alors que le pâturage les a fortement augmen-
Figure 8. Influence de la contamination butyrique sur l'ouverture des fromages.

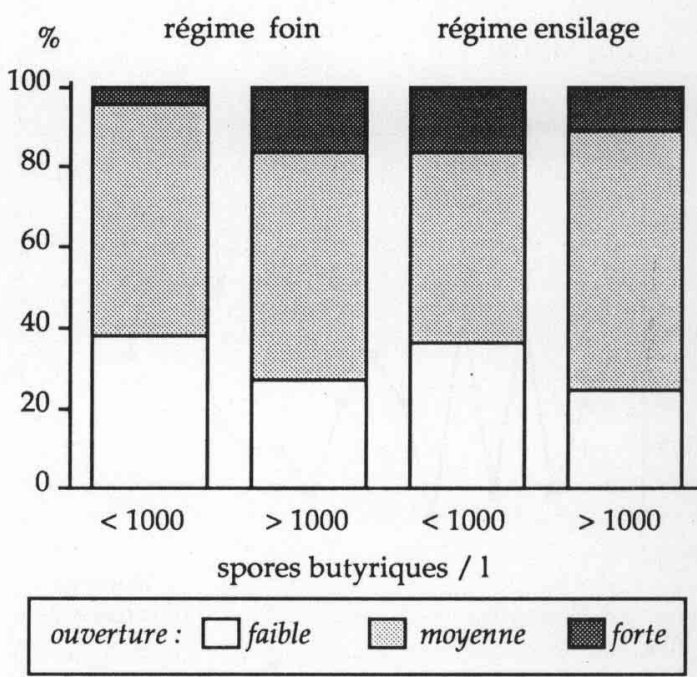

tées, en raison principalement des niveaux énergétiques différents de ces rations (Hoden et al 1985, Rémond 1985). Les différences observées entre les races utilisées dans cette étude confirment aussi les résultats antérieurs (taux butyreux plus élevé pour les vaches Frisonnes, et taux protéique plus élevé pour les Montbéliardes). Cependant, malgré des variations importantes de composition des laits utilisés pour la fabrication de fromage, (de 27 à $34 \mathrm{~g} / \mathrm{kg}$ de taux protéique) la méthode artisanale de fabrication employée a permis de réaliser un produit relativement homogène 24 heures après fabrication, du moins quant aux teneurs en matière sèche et en matière grasse.

Cette série d'essais confirme, pour ce type de fromage à pâte pressée, l'effet primordial du taux protéique du lait sur le rendement fromager. Les relations entre le rendement fromager et la composition du lait sont comparables à celles proposées par Banks et al (1981) ou Mietton (1987) avec d'autres conditions de conduite des troupeaux, et sur d'autres types de fabrications fromagères. L'influence du taux protéique sur la qualité des fromages affinés n'est pas apparue aussi nettement que pour le gruyère (Kerjean 1984), même si les fromages de meilleure qualité ont été obtenus avec des laits riches en matières grasses et protéiques, en 2ème partie de la lactation des animaux.

Dans nos conditions de fabrication particulières (sans refroidissement, ni conservation prolongée du lait), la qualité bactériologique du lait n'a pas influencé de façon importante la qualité du fromage. Toutefois, la contamination par les spores butyriques a été en moyenne relativement faible, inférieure à celle observée sur les laits produits avec de l'ensilage d'herbe dans des régions voisines (Coulon et Lilas 1988). Il n'en reste pas moins que les fromages fabriqués à partir des laits issus de régimes à base d'ensilage d'herbe, présentent plus de défauts (gonflements précoces, ouverture de la pâte), sans atteindre, toutefois l'ampleur des difficultés observées sur les fromages à pâte cuite (Bergere 1987). 
Cette étude a permis de mettre en évidence l'influence de facteurs zootechniques sur le rendement fromager et la qualité des fromages affinés. Ces résultats sont en accord avec ceux obtenus sur les premières étapes de la fabrication du fromage : effet de la race des vaches et du régime sur l'aptitude du lait à la coagulation (Froc et al 1988, Vertès et Hoden 1989, Vertès et al 1989). Ils permettent de mieux expliquer les variations saisonnières de rendement (liées au régime et au stade de lactation) ou d'une région à l'autre (effet de la race et du régime) (Olson 1977, Alistair 1983). Ils mettent en évidence les meilleures qualités fromagères des laits issus des régimes à base de foin ou d'herbe pâturée, avec toutefois de fortes variations au cours de l'année. L'effet de la race observé sur les fromages frais ne s'est pas répercuté sur les fromages affinés. La différence de composition des laits était d'ailleurs très faible par rapport aux comparaisons de race (Pie noire et Normande) citées par d'autres auteurs (Froc 1988, Vertès et al 1989).

La qualité des fromages affinés a été fortement influencée par la saison. Les défauts les plus fréquents sont apparus du mois de mars à juillet, en liaison, probablement, avec la méthode de fabrication artisanale, les difficultés d'affinage (surcharge des caves) et les changements de régime (mise à l'herbe). A cette même époque, un accroissement des défauts est d'ailleurs signalée sur Comté (Vassal et Grappin 1984), et sur Cheddar (Chapman et Burnet 1972).

Il est possible que la conduite de l'affinage puisse être adaptée au type de fromage selon l'origine des laits. En effet, les fromages provenant du lait d'ensilage semblent avoir une maturation plus rapide (souplesse, couleur, forme) que ceux issus de foin. Il en est de même pour les fromages provenant de lait de vaches Pie noires par rapport à ceux de vaches Montbéliardes.

Ces travaux mettent en évidence les difficultés de réalisation d'essais destinés à étudier les relations entre les qualités du lait et du fromage affiné, même avec un nombre important de fabrications fromagères complètes. Comme le souligne Kerjean (1984), la connaissance du fromage frais n'est généralement pas suffisamment précise pour prévoir la qualité du fromage affiné avec des réponses aux traitements expérimentaux parfois divergentes selon le critère considéré (aspect, pâte, goût). Ceci confirme l'intérêt de prolonger les études jusqu'au fromage affiné.

Même si les facteurs zootechniques pris indépendamment ont un effet limité, ils sont souvent cumulés dans les régions de montagne de l'Auvergne : ensilage d'herbe, sous-alimentation, génotype holstein. L'accumulation de ces conditions peut provoquer des baisses et des variations sensibles de la qualité fromagère des laits, avec un effet sans doute plus direct sur la fabrication d'Appellation d'Origine Contrôlée.

\section{Remerciements}

Nous tenons à remercier M. Verlaguet (Syndicat du St Nectaire Fermier) pour son aide et ses conseils lors de la fabrication et le jugement des fromages, et nos collègues du Laboratoire de Recherches fromagères d'Aurillac, $G$. Pradel et $\mathrm{R}$. Didienne, pour leur participation à cette étude.

\section{Références bibliographiques}

BANKS J.M., BANKS W., MUIR D.D., WILSON A.G., 1981. Cheese yield: composition does matter, Dairy Industries International, 46, 15-22.

BERGERE J.L, 1987. Les bactéries anaérobies sporulées du lait clostridium tyrobutyricum. In "Le lait matière première de l'industrie laitière ». 201 - 206.

CHAPMAN H. R., BURNETT J., 1972. Seasonal changes in the physical properties of milk for cheesemaking. Dairy industries, 37, 207-211.

COULON J.B., BINET M., 1987. Facteurs de variations du taux protéique du lait de vache en exploitation. Etude dans l'aire de ramassage de la coopérative fromagère agricole de Laguiole (Aveyron). Bull. Tech. C.R.Z.V. Theix, INRA, 68, 11-18

COULON J.B., LILAS J.P., 1988. Composition chimique et contamination butyrique du lait. Facteurs de variation dans le département de la Haute-Loire. INRA Prod. Anim., 1, 201-207.

COULON I B GAREL IP, HODEN A., JOURNET $M$., LIENARD G., 1985. Production laitière en zone de montagne : effets pluriannuels du type de ration hivernale et du niveau de complémentation. Bull. Tech. C.R.Z.V. Theix, INRA 61, 31-48.

DULPHY J.P., GAREL J.P., ANDRIEU J.P., ROUEL J., 1984. Intérêt du ressuyage avant la récolte d'ensilages d'herbe destinés à des vaches laitières. Bull. Tech. C.R.Z.V. Theix, INRA, 55, 17-23.

E.D.E. Haute-Saône et I.T.G., 1985. Incidence de la conduite alimentaire et des périodes de vêlages des vaches laitières sur les chutes saisonnières du taux protéique du lait.
FROC J., GLIBERT J., DALIPHAR T., DURAND P., 1988. Composition et qualité technologique des laits des vaches normandes et pie-noires. Effet de la race. INRA Prod. Anim., 1, 171-177.

GRANDISON A S ANDERSON M FORD G.D. NEWELL L., 1985. Interrelationships between the diet fed to cows, composition and properties of milk composition and quality of cheshire cheese from farmhouse manufacturers. I. Dairy Res., 52, 587-593.

HODEN A., COULON I.B., DULPHY J.P., 1985. Influence de l'alimentation sur la composition du lait III. - Effets des régimes alimentaires sur les taux butyreux et protéique. Bull. Tech. C.R.Z.V. Theix, INRA, 62, 69-79.

JOURNET M., CHILLIARD Y., 1985. Influence de l'alimentation sur la composition du lait. I. - Taux butyreux facteurs généraux. Bull. Tech. C.R.Z.V. Theix, INRA, 60, 12-23.

KERIEAN J R, 1984. La composition chimique du lait et ses incidences technologiques. Dans " La composition chimique du lait et ses incidences technologiques ». Colloque INRA-ENSA-INAPG-Rennes, septembre 1984.

MIETTON B., 1987. La préparation des laits de fromages en technologie pâtes molles - Revue des ENIL, 113, 2233.

OLSON N., 1977. Factors affecting cheese yields. Dairy industries International, 14, 19

REMOND B., 1985. Influence de l'alimentation sur la composition du lait de vache. II. - Taux protéique : facteurs généraux. Bull. Tech. C.R.Z.V. Theix, INRA, 62, 5367.

VERTES C., HODEN A., 1989. Qualité fromagère des laits de vaches en fonction du régime à base d'herbe. Le lait, $69,197-209$. 
VERTES C., HODEN A., GALLARD Y., 1989. Effet du niveau d'alimentation sur la composition chimique et la qualité fromagère du lait de vaches Holstein et Normandes. INRA, Prod. Anim., 2, 89-96.

VASSAL L., GRAPPIN R., 1984. Principaux problèmes rencontrés en industrie laitière en rapport avec la qualité des laits. Dans « La composition du lait et ses incidences technologiques ». Colloque INRA-ENSA-INAPG-Rennes, Septembre 1984

\section{Summary}

Effect of feeding and breed of dairy cows on Saint-Nectaire cheese making.

The purpose of this study, composed of 4 successive trials, was to analyse the related effects of different diets (based on hay, grass silage or pasture) and breeding factors (Friesian or Montbeliarde breed) on cheese making quality. The cheese manufactured was SaintNectaire cheese, a traditional French cheese. Fresh and ripe cheese yield were directly asso- ciated with differences in milk composition (mainly protein content) between breed and between diets. The highest cheese yield was obtained with hay diet and pasture diet compared to grass silage diet, and with Montbeliarde cows compared with Friesian cows. Breed has no great influence on ripe cheese quality. Cheese flavour remained unchanged between diets. Grass silage diet increased cheese openness and early blowing frequency but improved springiness and yellow color of the cheeses. The simple criteria used in this study to characterize cheese evolution during ripening were insuffisant to establish relations between fresh and ripe cheese quality. Nethertheless, they showed the importance of the cheese ripening room.

GAREL J.P., COULON J.B. 1990. Effet de l'alimentation et de la race des vaches sur la fabrication de fromage d'Auvergne de Saint-Nectarie. INRA Prod. Anim., 3 (2), 127-136.
Annexe 1

Grille de notation des fromages utilisés en 1979 et 1980

\begin{tabular}{|c|c|c|}
\hline Critères & Caractéristiques & Critère synthétique \\
\hline \multirow{2}{*}{ Forme } & Fromage bien formé & \\
\hline & Fromage déformé & Défauts de forme \\
\hline \multirow{2}{*}{ Pâte souplesse } & $\begin{array}{l}\text { Pâte souple de façon uniforme } \\
\text { Pâte souple par places }\end{array}$ & \\
\hline & $\begin{array}{l}\text { Pâte dure à très dure } \\
\text { Pâte coulante }\end{array}$ & Défauts de pâte \\
\hline \multirow{2}{*}{ Pâte ouverture } & $\begin{array}{l}\text { Ouverture faible } \\
\text { Ouverture moyenne }\end{array}$ & \\
\hline & Ouverture forte & Défauts d'ouverture \\
\hline Pâte couleur & $\begin{array}{l}\text { Blanc } \\
\text { Jaune pâle } \\
\text { Jaune }\end{array}$ & \\
\hline \multirow{2}{*}{ Goût } & $\begin{array}{l}\text { Goût et odeur agréable } \\
\text { Goût plat } \\
\text { Sans odeur étrangère }\end{array}$ & \\
\hline & $\begin{array}{l}\text { Odeur étrangère - amertume - rance } \\
\text { - excès de sel }\end{array}$ & Défauts de goût \\
\hline
\end{tabular}

Annexe 2

Grille de notation des fromages utilisés en 1981 et 1982. (Extrait du règlement intérieur de la Commission de contrôle de l'A.O.C

Saint-Nectaire 25.09.80).

\begin{tabular}{|c|c|c|c|}
\hline Critères & Caractéristiques & Note & Critère synthétique \\
\hline \multirow{2}{*}{$\begin{array}{l}\text { Forme et tenue } \\
\qquad\left(\begin{array}{l}0 \text { à } 5)\end{array}\right.\end{array}$} & $\begin{array}{l}\text { Forme régulière } \\
\text { Légère déformation }\end{array}$ & $\begin{array}{l}4-5 \\
3-4\end{array}$ & \\
\hline & Déformation & $<3$ & Défauts de forme \\
\hline \multirow[t]{2}{*}{$\begin{array}{l}\text { Pâte } \\
\text { (0 à } 5)\end{array}$} & $\begin{array}{l}\text { Pâte souple et onctueuse } \\
\text { - quelques ouvertures } \\
\text { - coloration homogène } \\
\text { Pâte ferme ou trop souple } \\
\text { - ouvertures plus } \\
\text { nombreuses }\end{array}$ & $4-5$ & \\
\hline & $\begin{array}{l}\text { Pâte cassante } \\
\text { - crayeuse - coulante } \\
\text { - spongieuse }\end{array}$ & $<3$ & Défauts de pâte \\
\hline \multirow[t]{2}{*}{$\begin{array}{c}\text { Goût - Odeur } \\
\left(\begin{array}{l}0 \text { à 10) }\end{array}\right.\end{array}$} & $\begin{array}{l}\text { Goût franc et savoureux } \\
\text { légèrement lactique } \\
\text { Goût plat sans odeur } \\
\text { étrangère }\end{array}$ & $\begin{array}{l}8-10 \\
6-8\end{array}$ & \\
\hline & Amertume - rance & $<6$ & Défauts de goût \\
\hline
\end{tabular}

